\title{
Cortisol Stress Biosensor Based on Molecular Imprinted Polymer ${ }^{\dagger}$
}

\author{
Supannika Klangphukhiew ${ }^{1}$, Roongnapa Srichana ${ }^{2}$ and Rina Patramanon $1,3, *$ \\ 1 Department of Biochemistry, Faculty of Science, Khon Kaen University, Khon Kaen 40002, Thailand; \\ supannika.k@kkumail.com \\ 2 Molecular Recognition Materials Research Unit, Department of Pharmaceutical Chemistry, \\ Faculty of Pharmaceutical Sciences, Prince of Songkla University, Hatyai, Songkhla 90112, Thailand; \\ roongnapa.s@psu.ac.th \\ 3 Protein and Proteomics Research Center for Commercial and Industrial Purposes (ProCCI), \\ Khon Kaen University, Khon Kaen 40002, Thailand \\ * Correspondence: narin@kku.ac.th; Tel.: +66-433-429-11 \\ + Presented at the Eurosensors 2017 Conference, Paris, France, 3-6 September 2017.
}

Published: 16 August 2017

\begin{abstract}
The molecular imprinted polymer (MIP) technique was applied to receptors that could imitate the performance of natural anti-cortisol antibody with high stability. Cortisol-molecular imprinted polymer (cortisol-MIP) was obtained using the multi-step swelling and polymerization protocol with cortisol as a target molecule. The suitable ratio of methacrylic acid:acrylamide :cortisol which allowed the formation of $642.3 \pm 35.8 \mathrm{~nm}$ homogenous particles was 2:1:1. Cortisol-MIP was integrated to the sensor. It was coated on the disposable screen-printed carbon electrode (SPCE) for portable electrochemical analysis. The physical properties of Cortisol-MIP were characterized by means of electron microscope techniques. The binding characteristics were evaluated via covalent patterns changing in FTIR spectra which were related to voltammetry responses. The SPCE modified with cortisol-MIP was able to perform various detection ranges from 1.28 to $20 \mathrm{nM}$ and the high selectivity compared to three other glucocorticoid analogs.
\end{abstract}

Keywords: core shell molecular imprinted polymer (Cs-MIP); cortisol; screen printed carbon electrode (SPCE)

\section{Introduction}

A personal health sensor has been a significant contribution to the area of medical science and technology. One strategy to conduce people to live in a longer period of good health accompanying with a sustained well-being is the preventative medicine by regularly monitoring physical and psychological health [1]. Cortisol has been used as a well-known commercial stress biomarker. A homeostasis response to psychological stress is indicated by an increased level of cortisol produced in hypothalamus-pituitary-adrenal (HPA) axis [2,3]. Chronic psychological stress contributing to the high level of cortisol relates to several health problems. Cortisol is one of the key roles that is involved in stress response produced from zona fasciculata which can be found in biological samples; blood serum, urine, saliva, sweats and hair in different amounts detected by conventional chromatographic techniques, immunoassays and small electronic device called sensor [4]. The sensor is to detect events or changes in environments and then provides a response output. The specific detections of structures and superstructures are based on geometrical interactions which define both dimensions and proposed functional groups. These can generate a mimic-recognition site. Molecular imprinted polymer (MIP) is a technique offering artificial receptors by imprinting of unnatural polymers with a target molecule [5]. Core shell MIP particles have significant advantages in term of more imprinting sites on external surface and greater stability of the structure [6,7]. MIP can be achieved when the 
target is present as a template in polymerization process and the changing outputs can be determined using the transducer. MIP has been integrated into several types of transducer including optical sensors, surface plasmon resonance (SPR), quartz crystal microbalance (QCM) and the most widely used one, electrochemical sensors. Electrochemical detection is a suitable method to develop a miniature device with small electrode [8].

In this study, MIP particles were coated on screen-printed carbon electrode (SPCE) as shown in schematic (Figure 1). The electrode pattern included a carbon working electrode and a silver/silver chloride $(\mathrm{Ag} / \mathrm{AgCl})$ reference electrode. The size of SPCE was small that could be detected by handheld potentiostat, suitable to be developed the cortisol point-of-care detection.

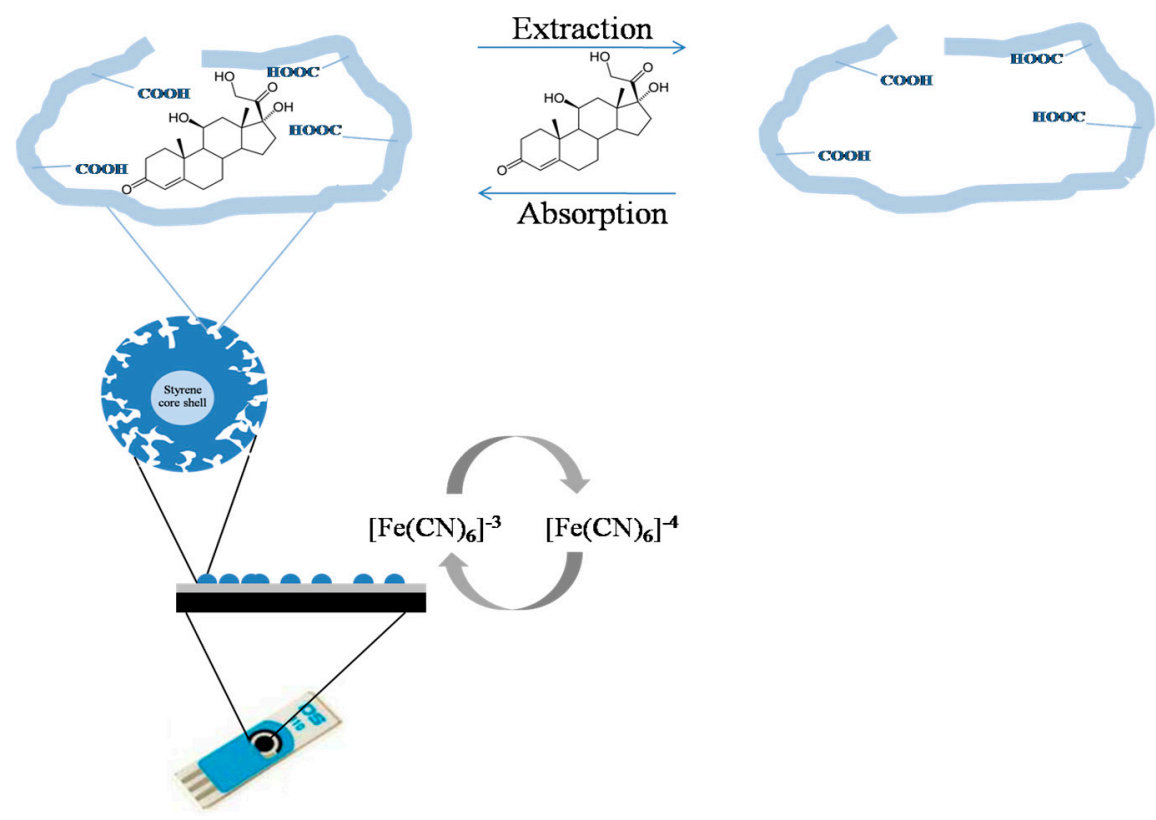

Figure 1. Schematic illustration of MIP particles modified screen printed carbon electrode detecting cortisol.

\section{Synthesis and Characterization of Cortisol-MIP Particles}

Cortisol-molecular imprinted polymer (cortisol-MIP) particles were obtained using the multi-step swelling and polymerization protocol with cortisol as a target molecule. Styrene bead was used as the core shell.The microemulsion was reacted by 2,2'-azobisbutyronitrile (AIBN; Aldrich Chemie, Milwaukee, WI, USA) with polyvinylalcohol in toluene solvent. Poly methacrylic acid-coethylene glycol dimethacrylate and $N, N^{\prime}$-(1,2-Dihydroxyethylene)-bis-acrylamide were polymerized with cortisol as template stirring at $70{ }^{\circ} \mathrm{C}$ under nitrogen stream for $24 \mathrm{~h}$. The cortisol template was washed by $10 \%$ acetic acid in methanol. The cortisol-MIP particles in suspension form were homogenized by High pressure homogenizer. The particle size was examined by Zetasizer. The suitable ratio of methacrylic acid:acrylamide:cortisol which allowed the formation of $642.3 \pm 35.8-\mathrm{nm}$ homogenous particles was 2:1:1. (Figure 2a). The surface of MIP was rougher than non-imprinted polymer (NIP; the similar reaction without cortisol template). The $4 \mu \mathrm{L}$ of Cortisol MIP suspension was dropped on screen-printed carbon electrode (SPCE) surface by $20 \%$ solgel mixture and incubated at $65^{\circ} \mathrm{C}$ overnight to fix the cortisol MIP particles on SPCE surface as shown in Figure $2 \mathrm{~b}$. 

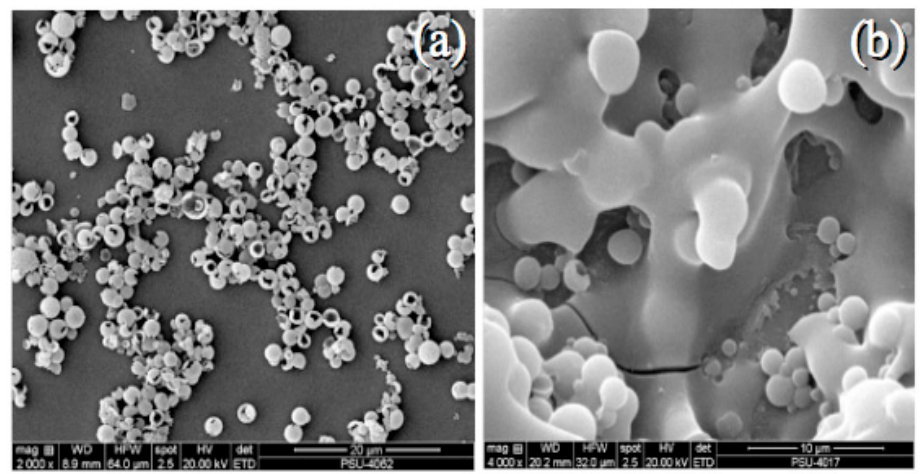

Figure 2. Scanning electron microscopic images of MIP after reducing the polymer sizes by $1 \times 10^{3}$ bars high pressure homogenizer (a) and coated on screen printed carbon electrode surface (b).

\section{Electrochemical Measurements}

Electrochemical measurements were performed by portable 910 PSTAT Mini (Metrohm autolab) connected with a computer system. The chromatogram was obtained by cyclic voltammetry $(\mathrm{CV})$ analysis in $5 \times 10^{-3} \mathrm{~mol} \cdot \mathrm{L}^{-1} \mathrm{~K}_{3}\left[\mathrm{Fe}(\mathrm{CN})_{6}\right] / \mathrm{K}_{4}\left[\mathrm{Fe}(\mathrm{CN})_{6}\right]$ in $1 \times 10^{-1} \mathrm{~mol} \cdot \mathrm{L}^{-1} \mathrm{KCl}$. CV was scanned in different potential values from $0.45 \mathrm{~V}$ to $-0.15 \mathrm{~V}$, at $0.02 \mathrm{Vs}^{-1}$ scan rate with 0.002 step of potential. The cyclic voltammograms of the SPCE modified with cortisol-MIP particles was indicated by the increase of redox peak at $0.2 \mathrm{~V}$. The detection range of cortisol concentrations was from 1.28 to $20 \mathrm{nM}$ with linear regression equation: $\Delta \mathrm{I}=1.92 \times \log$ [cortisol concentration] +13.72 at a correlation coefficient of 0.9804 (Figure 3). As the results of FTIR spectra, cortisol-MIP particles could rebind other cortisol molecules.

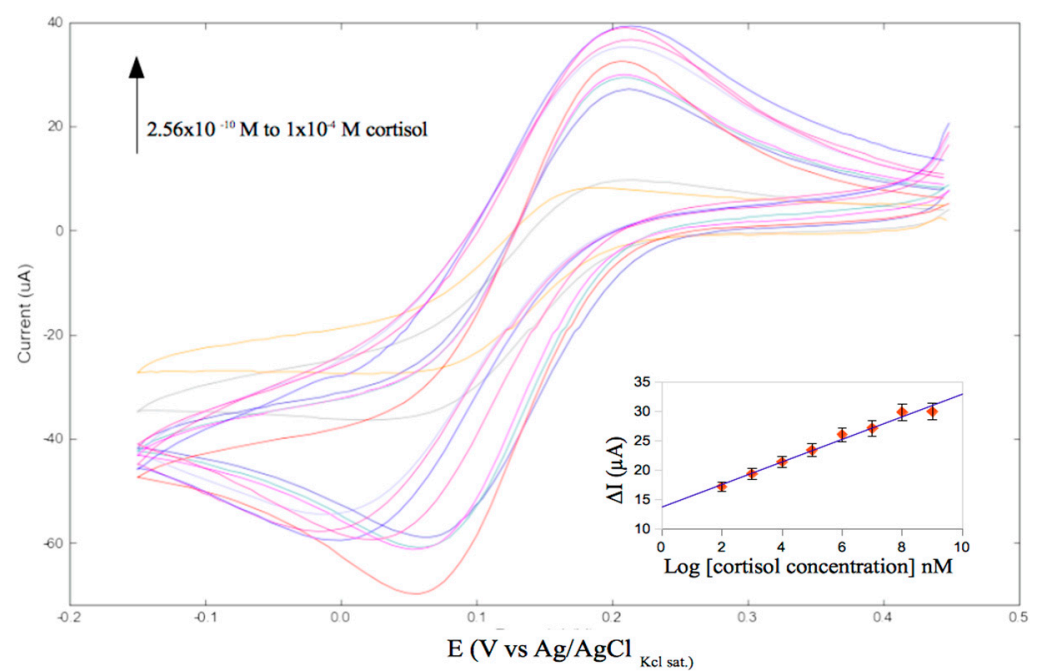

Figure 3. Cyclic voltammogram of MIP-modified SPCE responses to a wide range of cortisol concentrations from $1.28 \mathrm{nM}-20 \mu \mathrm{M}$. Measurements were performed in $5 \times 10^{-3} \mathrm{~mol} \cdot \mathrm{L}^{-1}$ $\mathrm{K}_{3}\left[\mathrm{Fe}(\mathrm{CN})_{6}\right] / \mathrm{K}_{4}\left[\mathrm{Fe}(\mathrm{CN})_{6}\right]$ in $1 \times 10^{-1} \mathrm{~mol} \cdot \mathrm{L}^{-1} \mathrm{KCl}$. CV was scanned in potential ranging from $-0.15 \mathrm{~V}$ to $0.45 \mathrm{~V}$ at $0.02 \mathrm{Vs}^{-1}$ scan rate with 0.002 step of potential.

\section{Summary}

This study revealed the achievement of core shell molecular imprinted polymer particles integrated on screen-printed carbon electrode to quantify the stress hormone, cortisol by hand held electrochemical device. The cortisol-MIP in particle form could enhance the surface area of cortisol binding cavities that allowed high performances of specificity and selectivity. The portable cortisol sensor exhibited $1.20 \mathrm{nM}$ in detection limit to recognize steroids. According to their structures with feasibility and cost-effectiveness, this biosensor could therefore be developed to use in point-of-care testing. 
Acknowledgments: This work has been funded by Development and Promotion for Science and Technology talents project (DPST) by the institute for the Promotion of Teaching Science and Technology. The authors would like to thank the Department of Pharmaceutical Chemistry, Faculty of Pharmaceutical Sciences, Prince of Songkla University for the facility.

Conflicts of Interest: The authors declare no conflict of interest.

\section{References}

1. Ding, S.; Schumacher, M. Sensor monitoring of physical activity to improve glucose management in diabetic patients: A review. Sensors 2016, 16, 589, doi:10.3390/s16040589.

2. Holsboer, F.; Ising, M. Stress hormone regulation: Biological role and translation into therapy. Annu. Rev. Psychol. 2010, 61, 81-109, doi:10.1146/annurev.psych.093008.100321.

3. Hellhammer, D.H.; Wust, S.; Kudielka, B.M. Salivary cortisol as a biomarker in stress research. Psychoneuroendocrinology 2009, 34, 163-171, doi:10.1016/j.psyneuen.2008.10.026.

4. Kaushik, A.; Vasudev, A.; Arya, S.K.; Pasha, S.K.; Bhansali, S. Recent advances in cortisol sensing technologies for point-of-care application. Biosens. Bioelectron. 2014, 53, 499-512, doi:10.1016/j.blos.2013.09.060.

5. Dickert, F.L.; Lieberzit, P.; Tortschanoff, M. Molecular imprints as artificial antibodies-a new generation of chemical sensor. Sens. Actuators B Chem. 2000, 65, 186-189, doi:S0925-4005-99-00327-5.

6. Paul, P.K.; Treetong, A.; Suedee, R. Biomimic insulin-imprinted polymer nanoparticles as a potential oral drug delivery system. Acta Pharm. 2017, 67, 149-168, doi:10.1515/acph-2017-0020.

7. Wackerlig, J.; Lieberzeit, P.A. Molecularly imprinted polymer nanoparticles in chemical sensing-Synthesis, characterization and application. Sens. Actuators B Chem. 2015, 207, 144-157, doi:10.1016/j.snb.2014.09.094.

8. Blanco-Lopez, M.C.; Lobo-Castanon, M.J.; Miranda-Ordirers, A.J.; Tunon-Blanco, P. Electrochemical sensors based on molecularly imprinted polymers. Trends Anal. Chem. 2004, 23, 36-48, doi:10.1016/s01659936(04)00102-5.

(C) 2017 by the authors; licensee MDPI, Basel, Switzerland. This article is an open access article distributed under the terms and conditions of the Creative Commons by Attribution (CC BY) license (http://creativecommons.org/licenses/by/4.0/). 